\title{
BMJ Open Effects of hyperuricaemia, with the superposition of being overweight and hyperlipidaemia, on the incidence of acute kidney injury following cardiac surgery: a retrospective cohort study
}

Yiqi Su (D) , ${ }^{1}$ Haoxuan $\mathrm{Li}^{2}$ Yang Li, ${ }^{3}$ Xialian $\mathrm{Xu},{ }^{3}$ Bo Shen, ${ }^{3}$ Wuhua Jiang, ${ }^{3}$ Yimei Wang, ${ }^{3}$ Yi Fang, ${ }^{3}$ Chunsheng Wang, ${ }^{4}$ Zhe Luo, ${ }^{5}$ Xiaoqiang Ding, ${ }^{3}$ Jie Teng,,${ }^{1,3}$ Jiarui $\mathrm{Xu}^{3}$

To cite: Su Y, Li H, Li Y, et al. Effects of hyperuricaemia, with the superposition of being overweight and hyperlipidaemia, on the incidence of acute kidney injury following cardiac surgery: a retrospective cohort study. BMJ Open 2022;12:e047090. doi:10.1136/ bmjopen-2020-047090

- Prepublication history and additional supplemental material for this paper are available online. To view these files, please visit the journal online (http://dx.doi.org/10.1136/ bmjopen-2020-047090).

YS and HL contributed equally. JT and JX contributed equally.

Received 20 November 2020 Accepted 23 November 2021

Check for updates

(C) Author(s) (or their employer(s)) 2022. Re-use permitted under CC BY-NC. No commercial re-use. See rights and permissions. Published by BMJ.

For numbered affiliations see end of article.

\section{Correspondence to}

Dr Jiarui Xu;

xu.jiarui@zs-hospital.sh.cn and

Dr Jie Teng;

teng.jie@zs-hospital.sh.cn

\section{ABSTRACT}

Objectives Acute kidney injury (AKI) is a common complication of cardiac surgery. This study aimed to explore the effects of hyperuricaemia, being overweight and hyperlipidaemia as risk factors for AKI in patients following cardiac surgery (cardiac surgery-associated acute kidney injury (CSA-AKI)).

Design Retrospective observational study.

Setting University teaching, grade-A tertiary hospital in Shanghai, China.

Participants Patients who underwent cardiac surgery from July 2015 to December 2015 in Zhongshan Hospital, Fudan University.

Main outcome measures We investigated the effect of hyperuricaemia, in combination with being overweight and hyperlipidaemia, on the risk of CSA-AKI.

Results A total of 1420 patients were enrolled. The AKI incidence in the highest uric acid group was $44.4 \%$, while that in the lowest uric acid group was $28.5 \%(p<0.001)$. Patients in the higher uric acid quartiles were more likely to be overweight and hyperlipidaemic at the same time $(p<0.001)$. Multivariate logistic regression analysis showed that hyperuricaemia was an independent risk factor for AKI (OR=1.237, 95\% Cl 1.095 to 1.885; $p=0.009)$; being overweight or hyperlipidaemia alone was not an independent risk factor, but the combination of being overweight and hyperlipidaemia was $(\mathrm{R}=1.544,95 \% \mathrm{Cl}$ 1.059 to $2.252 ; p=0.024)$. In the final model, the $\mathrm{OR}$ value increased to 3.126 when hyperuricaemia was combined with being overweight and hyperlipidaemia, and the Hosmer-Lemeshow test showed that all three models fit well ( $p=0.433,0.638$ and 0.597 , respectively).

Conclusions The combination of being overweight and having hyperlipidaemia was an independent risk factor, but being overweight or having hyperlipidaemia alone was not. The combination of hyperuricaemia, being overweight and hyperlipidaemia further increased the risk of CSA-AKI.

\section{BACKGROUND}

Acute kidney injury (AKI) is a common complication of cardiac surgery. The cardiac
Strengths and limitations of this study

- This article offers an exploration of the combination of risk factors on the incidence of acute kidney injury (AKI) in patients following cardiac surgery.

- Appropriate statistical methods were used to analyse the findings of the study.

- This was a retrospective and observational study, which may limit the generalisation of the conclusions.

- Further research needs to be conducted on whether reducing the combination of risk factors can reduce the risk of AKI.

surgery-associated acute kidney injury (CSAAKI) rate is approximately $20 \%-40 \%$, and the mortality rate is up to $30 \%$, which affects the long-term prognosis. ${ }^{2}$ CSA-AKI has many non-modifiable risk factors, including age, race and pre-existing comorbidities such as hypertension and chronic kidney disease (CKD); there are some potentially modifiable risk factors such as nephrotoxic medications, anaemia and fluid administration. ${ }^{3}$ Studies have shown that hyperuricaemia and increased body mass index (BMI) are independent risk factors for CSA-AKI ${ }^{4-6}$ that are modifiable for elective surgery. Interventions to control modifiable risk factors may be the main way to prevent CSA-AKI. ${ }^{7}$

Uric acid is the end product of purine metabolism and is excreted by the kidneys. While the kidneys excrete approximately $70 \%$ of uric acid, $90 \%-95 \%$ is reabsorbed in the renal tubule after being filtered by the glomerulus. Therefore, serum uric acid levels depend on glomerular filtration capacity and renal tubular reabsorption. Previous studies have confirmed that preoperative 
hyperuricaemia is an independent risk factor for CSAAKI. ${ }^{8-10}$ A meta-analysis including 18 cohort studies with 75200 patients found that patients with hyperuricaemia have a significantly higher risk of AKI compared with the control group (OR 2.24, 95\% CI 1.76 to $2.86 ; \mathrm{p}<0.01$ ). The mechanism of AKI caused by hyperuricaemia is related to the crystallisation of uric acid, which may also activate an epithelial-mesenchymal proinflammatory response.

Hyperuricaemia is often associated with metabolic disorders, such as being overweight and hyperlipidaemia. Studies have confirmed that serum uric acid level is positively correlated with serum total cholesterol (TC), triglycerides and low-density lipoprotein (LDL) levels and negatively correlated with high-density lipoprotein (HDL) levels. ${ }^{11}$ Other studies have suggested that hyperlipidaemia is a risk factor for AKI after surgery. ${ }^{12}$ In addition, the incidence of AKI can increase with increasing BMI. ${ }^{13} 14$ Previous studies also confirmed that preoperative serum creatinine (SCr) and uric acid levels increased with an increase in BMI, which was an independent risk factor for AKI. ${ }^{6}$

With economic development and changes in lifestyle and diet patterns, the risk factors for AKI in the Chinese population have increased rapidly in recent years. An increasing number of people tend to be obese or have hypertension, diabetes, hyperuricaemia and hyperlipidaemia. Considering that many risk factors might have additive effects and to provide a reference for clinicians, this study explored the relationship between hyperuricaemia, being overweight and hyperlipidaemia, and the effect of these factors on the risk of CSA-AKI.

\section{MATERIALS AND METHODS}

\section{Patient selection}

We retrospectively analysed data from patients who underwent cardiac surgery between July 2015 and December 2015 in our hospital. Patients were excluded if they were $<18$ years of age, had pre-existing end-stage renal disease, had kidney transplantation, intended to receive heart transplantation or had missing data

\section{Definitions}

AKI was defined according to the following Kidney Disease: Improving Global Outcomes (KDIGO) criteria $^{15}$ : (1) increase in SCr by $\geq 26.5 \mu \mathrm{mol} / \mathrm{L}$ within 48 hours; (2) increase in SCr to $\geq 1.5$ times baseline, which is known or presumed to have occurred within the prior 7 days; and (3) urine volume of $<0.5 \mathrm{~mL} / \mathrm{kg} /$ hour for 6 hours and staged according to the SCr and urine output. Severe AKI was defined as having KDIGO stage 2 or 3. Hypertension was defined as having a systolic blood pressure (BP) of $\geq 140 \mathrm{~mm} \mathrm{Hg}$ or a diastolic BP of $\geq 90 \mathrm{~mm} \mathrm{Hg}$ or an individual currently using antihypertensive drugs. ${ }^{16} \mathrm{Hy}-$ peruricaemia was defined as having serum uric acid levels of $>360 \mu \mathrm{mol} / \mathrm{L}$ (women) and $>420 \mu \mathrm{mol} / \mathrm{L}$ (men and postmenopausal women). Hyperlipidaemia was defined as having levels of triglyceride of $\geq 1.70 \mathrm{mmol} / \mathrm{L}$ and high-density lipoprotein cholesterol (HDL-c) of $<1.04 \mathrm{mmol} / \mathrm{L}$ (and/or) TC of $\geq 5.18 \mathrm{mmol} / \mathrm{L}$. Being overweight was defined as having a BMI of $\geq 24 \mathrm{~kg} / \mathrm{m}^{2}{ }^{2}{ }^{7}$ High-risk surgery was defined as either aortic surgery, macrovascular surgery or combined surgery. CKD was defined as having an estimated glomerular filtration rate (eGFR) of $<60 \mathrm{~mL} /\left(\mathrm{min} / 1.73 \mathrm{~m}^{2}\right)$.

\section{Data collection and groups}

Preoperative information of all patients was collected, including sex, age, preoperative comorbidities (hypertension and diabetes), New York Heart Association (NYHA) cardiac function classification grade, SCr, eGFR, blood glucose and blood lipid levels. Intraoperative data included surgical type, extracorporeal circulation time and aortic clamping time. Postoperative data included length of hospital stay, length of intensive care unit (ICU) stay and mechanical ventilation days. Patients were divided into AKI versus non-AKI groups, and hyperuricaemia versus normal uric acid groups. The uric acid levels were categorised into four groups according to the quartiles of gender-specific distribution: for men: Q1, $<311 \mu \mathrm{mol} / \mathrm{L}$; Q2, 311-366 $\mu \mathrm{mol} / \mathrm{L}$; Q3, 366-442 $\mu \mathrm{mol} / \mathrm{L}$; Q4, >442 $\mu \mathrm{mol} / \mathrm{L}$; for women: Q1, <246 $\mathrm{mol} / \mathrm{L} ; \mathrm{Q} 2$, 246-304 $\mu \mathrm{mol} / \mathrm{L}$; Q3, 304-369 $\mathrm{mol} / \mathrm{L} ; \mathrm{Q} 4,>369 \mu \mathrm{mol} / \mathrm{L}$. Poor short-term outcomes include death during hospitalisation or discharge from the hospital automatically after abandoning treatment.

\section{Patient and public involvement}

The patients and the public were not involved in the design, conduct, reporting or dissemination plans of this research.

\section{Statistical analysis}

Statistical analysis was performed using SPSS V.20.0. The independent sample t-test or the Mann-Whitney test was used to compare continuous variables, and dichotomous variables were analysed using the $\chi^{2}$ test or Fisher's exact test. Pearson's correlation was used to analyse the relationship between uric acid, blood lipid and BMI. A logistic regression model was used to analyse the risk factors for AKI. We first performed univariate analysis of all variables, then the backward stepwise selection method was used for multiple regression analysis, with a $p$ value of $<0.05$ as the inclusion criterion and $p$ value of $\geq 0.10$ or higher as the exclusion criterion. The Hosmer-Lemeshow test was used to verify the goodness of fit of the models. Statistical significance was set at a $\mathrm{p}$ value of $<0.05$.

\section{RESULTS}

\section{Baseline characteristics}

We identified 1420 patients who underwent cardiac surgery with a mean age of $57.1 \pm 12.2$ years. The baseline demographic and medical characteristics for all patients, as divided by the uric acid quartiles, are shown in table 1 . A history was documented for hypertension in 532 
Table 1 Baseline characteristics of the study population according to uric acid quartiles

\begin{tabular}{|c|c|c|c|c|c|c|}
\hline & All (=1420) & Q1 ( $n=351)$ & Q2 (n=360) & Q3 (n=353) & Q4 ( $n=356)$ & $P$ value \\
\hline Gender (male), n (\%) & $833(58.7)$ & $127(36.2)$ & $207(57.5)$ & $231(65.4)$ & $272(76.4)$ & $<0.001$ \\
\hline Age (years) & $57.1 \pm 12.2$ & $56.7 \pm 12.5$ & $56.9 \pm 12.3$ & $56.9 \pm 12.4$ & $57.7 \pm 11.5$ & 0.877 \\
\hline Overweight, n (\%) & $601(42.3)$ & $108(30.8)$ & $131(36.4)$ & $170(48.2)$ & 192 (53.9) & $<0.001$ \\
\hline Hypertension, n (\%) & $532(37.5)$ & $113(32.2)$ & $127(35.3)$ & $141(39.9)$ & $151(42.4)$ & 0.023 \\
\hline Blood glucose (mmol/L) & $5.2 \pm 0.7$ & $5.4 \pm 1.8$ & $5.3 \pm 1.7$ & $5.3 \pm 1.4$ & $5.3 \pm 1.4$ & 0.788 \\
\hline Glycosylated haemoglobin (\%) & $5.8 \pm 0.5$ & $6.1 \pm 0.7$ & $5.6 \pm 0.2$ & $5.8 \pm 0.5$ & $5.7 \pm 0.5$ & 0.735 \\
\hline Blood urea nitrogen (mmol/L) & $6.0 \pm 2.0$ & $5.2 \pm 1.5$ & $5.7 \pm 1.5$ & $6 \pm 1.8$ & $7.2 \pm 2.6$ & $<0.001$ \\
\hline Serum creatinine $(\mu \mathrm{mol} / \mathrm{L})$ & $80.8 \pm 22.4$ & $67.7 \pm 15.3$ & $76.6 \pm 14.6$ & $82.1 \pm 16.6$ & $96.6 \pm 29.1$ & $<0.001$ \\
\hline eGFR $\left(\mathrm{mL} /\left(\mathrm{min} / 1.73 \mathrm{~m}^{2}\right)\right)$ & $86.4 \pm 20.6$ & $97.3 \pm 20.1$ & $89.1 \pm 18.1$ & $84.7 \pm 18.5$ & $74.7 \pm 19.3$ & $<0.001$ \\
\hline Cholesterol (mmol/L) & $4 \pm 0.9$ & $4 \pm 0.9$ & $4 \pm 0.9$ & $4 \pm 0.9$ & $4 \pm 0.9$ & 0.073 \\
\hline Triglyceride (mmol/L) & $1.3 \pm 0.8$ & $1.1 \pm 0.6$ & $1.3 \pm 0.7$ & $1.5 \pm 1$ & $1.5 \pm 0.9$ & $<0.001$ \\
\hline Low-density lipoprotein cholesterol (mmol/L) & $2.3 \pm 0.8$ & $2.2 \pm 0.8$ & $2.2 \pm 0.8$ & $2.4 \pm 0.8$ & $2.3 \pm 0.8$ & 0.132 \\
\hline High-density lipoprotein cholesterol (mmol/L) & $1.2 \pm 0.3$ & $1.3 \pm 0.4$ & $1.2 \pm 0.3$ & $1.1 \pm 0.3$ & $1.1 \pm 0.4$ & $<0.001$ \\
\hline Hyperlipidaemia, n (\%) & $292(20.6)$ & $36(10.3)$ & 68 (18.9) & $86(24.4)$ & $102(28.7)$ & $<0.001$ \\
\hline Albumin (g/L) & $40.8 \pm 3.9$ & $39.1 \pm 3.4$ & $39.7 \pm 3.3$ & $40.1 \pm 3.6$ & $40.4 \pm 3.8$ & $<0.001$ \\
\hline Urolithiasis & $76(5.4)$ & $15(4.3)$ & $22(6.1)$ & $20(5.7)$ & $19(5.3)$ & 0.287 \\
\hline Urinary tract infection & $30(2.1)$ & $7(2.0)$ & $7(1.9)$ & $8(2.3)$ & $8(2.2)$ & 0.766 \\
\hline Nephrotoxic drugs & $141(9.9)$ & $32(9.1)$ & 32 (8.9) & 37 (10.5) & $40(11.2)$ & 0.021 \\
\hline
\end{tabular}

Results presented as mean \pm SD or number (percentage).

eGFR, estimated glomerular filtration rate.

patients $(37.5 \%)$ and diabetes in 221 patients $(15.6 \%)$. When analysed by the quartile of the uric acid level, the patients with higher uric acid levels were more likely to be older, with more occurrences of hypertension, higher BMI and levels of blood urea nitrogen, SCr, albumin and triglycerides. There were no significant differences in cholesterol, low-density lipoprotein cholesterol (LDLc), glycosylated haemoglobin and blood glucose levels among the four groups. Moreover, patients in the higher uric acid quartiles were more likely to be overweight and to have hyperlipidaemia (all $\mathrm{p}<0.001$ ). In contrast, the patients in the higher uric acid quartiles showed lower levels of eGFR and HDL-c (all $\mathrm{p}<0.001)$ (table 1$)$. We also found significant differences between the AKI group and the non-AKI group for male sex, age, diabetes, NYHA grade $>$ II, hyperuricaemia, preoperative $\mathrm{SCr}$ and uric acid levels (all $\mathrm{p}<0.001)$ (online supplemental table 1 ). A total of 117 patients $(8.2 \%)$ were diagnosed with CKD. Compared with the non-AKI group, the AKI group also had a higher proportion of patients with CKD $(11.8 \%$ vs $6.2 \%, \mathrm{p}<0.001)$. The AKI group had a higher proportion of being overweight (47.9\% vs $39.1 \%, \mathrm{p}=0.006)$ and higher BMI $(24.1 \pm 3.5$ vs $23.4 \pm 3.1, \mathrm{p}<0.001)$. There were no significant differences in levels of cholesterol, triglycerides and LDL-c between the two groups (online supplemental table 1). There was no significant difference in extracorporeal membrane oxygenation support and the use of inotropic agents between the two groups, but the AKI group had a higher proportion of using nephrotoxic drugs $(20.3 \%$ vs $4 \%, \mathrm{p}<0.001)$, diuretics $(97.1 \%$ vs $93.2 \%, p=0.002)$ and vasoactive agents $(93.2 \%$ vs $89.4 \%$, $\mathrm{p}=0.015$ ). The AKI group also had a higher proportion of 24-hour fluid balance ( 1.2 vs $0.3, \mathrm{p}<0.001)$ and the incidence of low cardiac output syndrome (LCOS; $19.7 \%$ vs $8.4 \%, \mathrm{p}<0.001$; online supplemental table 1 ).

\section{AKI incidence and short-term outcomes}

When analysed by quartile of the uric acid level, the AKI incidence correlated with higher uric acid levels $(\mathrm{p}<0.001)$. The patients in the higher uric acid quartiles displayed longer lengths of hospital stay and ICU stay (all $\mathrm{p}<0.001$ ) (table 2). However, the incidence of severe AKI, renal replacement therapy (RRT) treatment and poor short-term prognosis showed no statistical differences among the uric acid quartiles (all p >0.05) (table 2). 
Table 2 Comparison of AKI incidence and short-term outcomes according to uric acid quartiles

\begin{tabular}{|c|c|c|c|c|c|}
\hline & Q1 $(n=351)$ & Q2 $(n=360)$ & Q3 $(n=353)$ & Q4 (n=356) & $P$ value \\
\hline AKI, n (\%) & $100(28.5)$ & $116(32.2)$ & $144(40.8)$ & $158(44.4)$ & $<0.001$ \\
\hline Severe AKI, n (\%) & $21(6.0)$ & $26(7.2)$ & $28(7.9)$ & $33(9.3)$ & 0.094 \\
\hline Length of hospital stay (days) & $13.5 \pm 15.8$ & $13.2 \pm 6.9$ & $13.2 \pm 5.1$ & $14.2 \pm 6.8$ & $<0.001$ \\
\hline Length of intensive care unit stay (hours) & $46.6 \pm 80.3$ & $46.4 \pm 76.4$ & $53.1 \pm 74.7$ & $64.3 \pm 116$ & $<0.001$ \\
\hline
\end{tabular}

Results presented as mean \pm SD or number (percentage). $\mathrm{AKI}$, acute kidney injury.

When grouped by AKI and non-AKI, the patients with AKI required longer ICU and hospital stays (all $p<0.001$, online supplemental table 1); the incidence of poor short-term outcomes in the AKI group was significantly higher than that in the non-AKI group $(3.3 \%$ vs $0.2 \%$, $\mathrm{p}<0.001$; online supplemental table 1 ).

When grouped by hyperuricaemia, the incidence of AKI, severe AKI and RRT treatment in the hyperuricaemia group was significantly higher than that in the normal uric acid group (44.2\% vs 33\%, $\mathrm{p}<0.001 ; 10.7 \%$ vs $6.2 \%, \mathrm{p}=0.003$; and $3.2 \%$ vs $1.4 \%, \mathrm{p}=0.027$ ) (online supplemental table 2).

The length of ICU stay in the hyperuricaemia group was significantly higher than that in the normal uric acid group $(\mathrm{p}<0.001)$ (online supplemental table 2$)$.

\section{Correlation analysis between uric acid, lipids, BMI and glucose}

Pearson correlation analysis showed the uric acid level is positively correlated with the triglyceride level $(r=0.221$, $\mathrm{p}<0.001)$ and BMI $(\mathrm{r}=0.157, \mathrm{p}<0.001)$, respectively. There was a negative correlation between uric acid and HDL-c levels $(\mathrm{r}=0.190, \mathrm{p}<0.001)$. There was no correlation between the uric acid level and the level of cholesterol $(\mathrm{r}=0.014, \mathrm{p}=0.603)$, LDL-c $(\mathrm{r}=0.044, \mathrm{p}=0.095)$ or blood glucose $(\mathrm{r}=-0.016, \mathrm{p}=0.549)$ (figure 1$)$. The AKI incidence in different subgroups showed that the combination of hyperuricaemia, hyperlipidaemia and being overweight had the highest AKI incidence (56.7\%); this was followed by hyperuricaemia combined with being overweight, and hyperuricaemia acid combined with hyperlipidaemia, with AKI incidences of $50.5 \%$ and $49.2 \%$, respectively (figure 2).

\section{Logistic regression analysis of risk factors for CSA-AKI}

Univariate logistic analysis was conducted and screened out several risk factors for AKI, including male sex $(\mathrm{OR}=1.524,95 \% \mathrm{CI} 1.219$ to 1.905$)$, age $(\mathrm{OR}=1.022$, $95 \%$ CI 1.013 to 1.032$)$, being overweight ( $\mathrm{OR}=1.403$, $95 \%$ CI 1.100 to 1.790$)$, diabetes (OR=1.353, 95\% CI 1.011 to 1.811), preoperative coronary angiography $(\mathrm{OR}=1.290$, $95 \%$ CI 1.039 to 1.602$)$, NYHA grade $>$ II $(\mathrm{OR}=1.375$, $95 \% \mathrm{CI} 1.043$ to 1.812 ), preoperative eGFR $<60 \mathrm{~mL} /$ $\left(\mathrm{min} / 1.73 \mathrm{~m}^{2}\right) \quad(\mathrm{OR}=2.016,95 \% \mathrm{CI} 1.379$ to 2.949$)$, albuminuria (OR=1.394, 95\% CI 1.024 to 2.203), hyperuricaemia ( $\mathrm{OR}=1.696,95 \%$ CI 1.275 to 2.022$)$, high-risk surgery $(\mathrm{OR}=2.065,95 \% \mathrm{CI} 1.538$ to 2.772$)$, extracorporeal cardiopulmonary bypass $(\mathrm{CPB})$ time $(\mathrm{OR}=1.010$, $95 \%$ CI 1.007 to 1.014 ), LCOS (OR=2.241, 95\% CI 1.183 to 3.576$)$ and aortic occlusion time $(\mathrm{OR}=1.015,95 \% \mathrm{CI}$ 1.010 to 1.020 ) (table 3 ).

Multiple logistic regression analysis showed that hyperuricaemia was an independent risk factor for CSA-AKI $(\mathrm{OR}=1.237,95 \%$ CI 1.095 to $1.885 ; \mathrm{p}=0.009)$ in model 1 , after adjusting for the confounding factors of male sex, age, being overweight, diabetes, angiography, NYHA >II, preoperative eGFR $<60 \mathrm{~min} / 1.73 \mathrm{~m}^{2}$, high-risk surgery, extracorporeal $\mathrm{CPB}$ time and aortic occlusion time. When the combination of being overweight and hyperlipidaemia was included in model 2 , it became a risk factor for AKI (OR=1.544, 95\% CI 1.059 to 2.252; $\mathrm{p}=0.024$ ), while hyperuricaemia became insignificant. In model 3 , adjusting for confounding factors, we found that the combination of hyperuricaemia, being overweight and hyperlipidaemia further increased the risk of postoperative AKI (OR=3.126, 95\% CI 1.731 to $5.646 ; \mathrm{p}<0.001)$. The Hosmer-Lemeshow test showed that all models fit well $(\mathrm{p}=0.433,0.638$ and 0.597 , respectively) (table 4$)$.

\section{DISCUSSION}

This study found that patients with higher uric acid levels had a higher incidence of AKI, and these patients were more commonly overweight and hyperlipidaemic at the same time. Hyperuricaemia was positively correlated with triglycerides and BMI; it was negatively correlated with HDL-c. Hyperuricaemia was an independent risk factor for CSA-AKI (OR=1.237, 95\% CI 1.095 to $1.885 ; \mathrm{p}=0.009)$; the combination of being overweight and having hyperlipidaemia was an independent risk factor, but being overweight or having hyperlipidaemia alone were not. The combination of hyperuricaemia, being overweight and hyperlipidaemia further increases the risk of AKI.

The incidence of AKI increased according to the uric acid quartile, and patients with higher uric acid levels displayed longer lengths of hospital stay and ICU stay. The level of uric acid is related to blood lipids and BMI, 

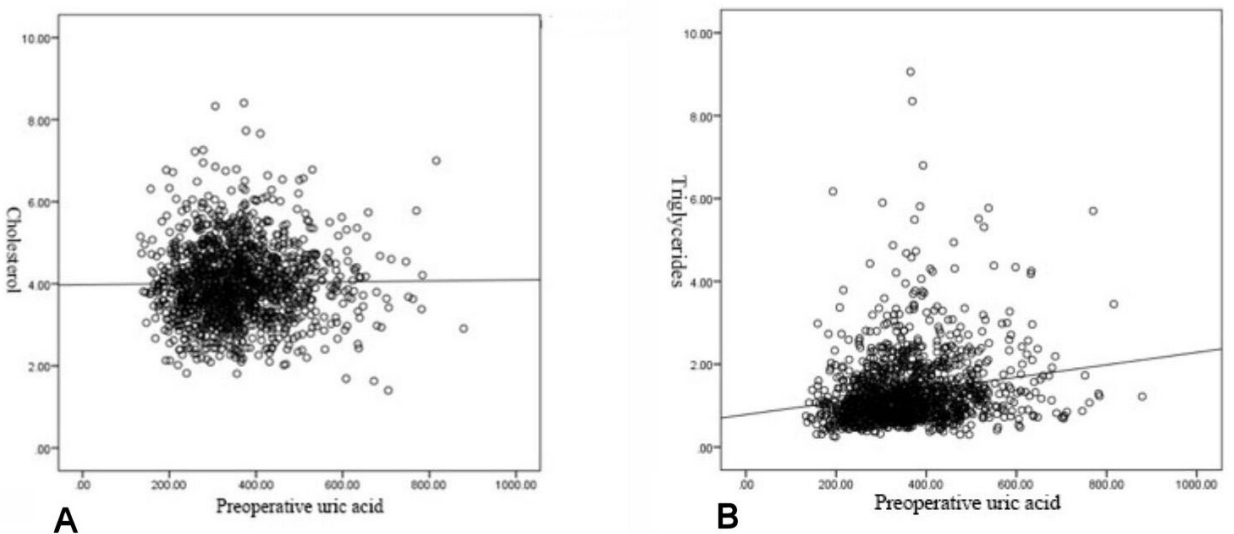

$r=0.014$

$r=0.221$

$P=0.603$

$P<0.001$
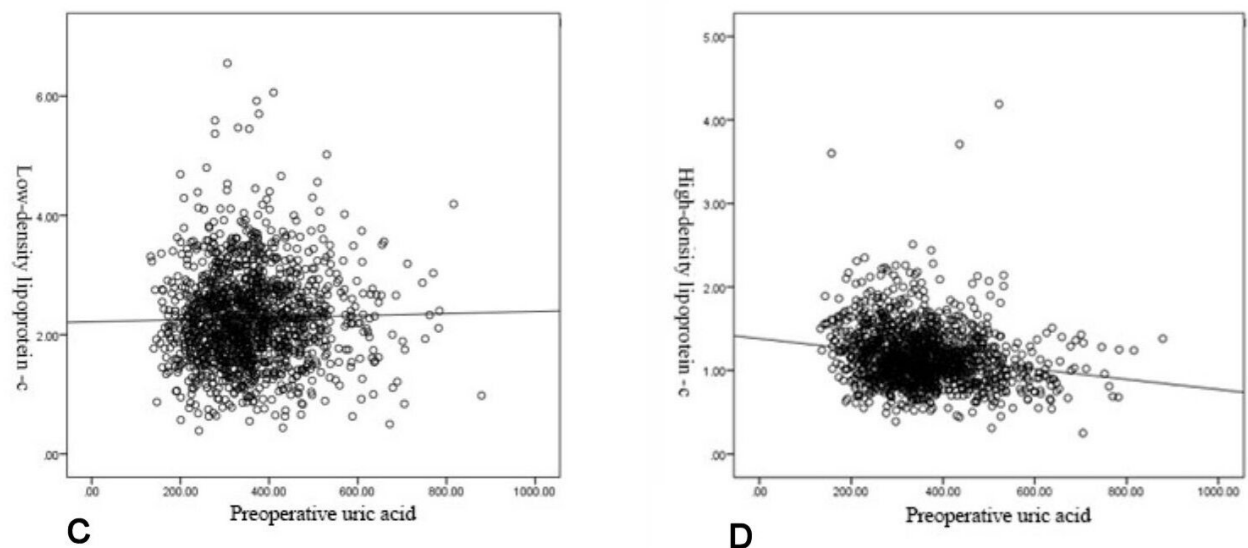

$r=0.044$

$r=-0.190$

$P=0.095$

$P<0.001$
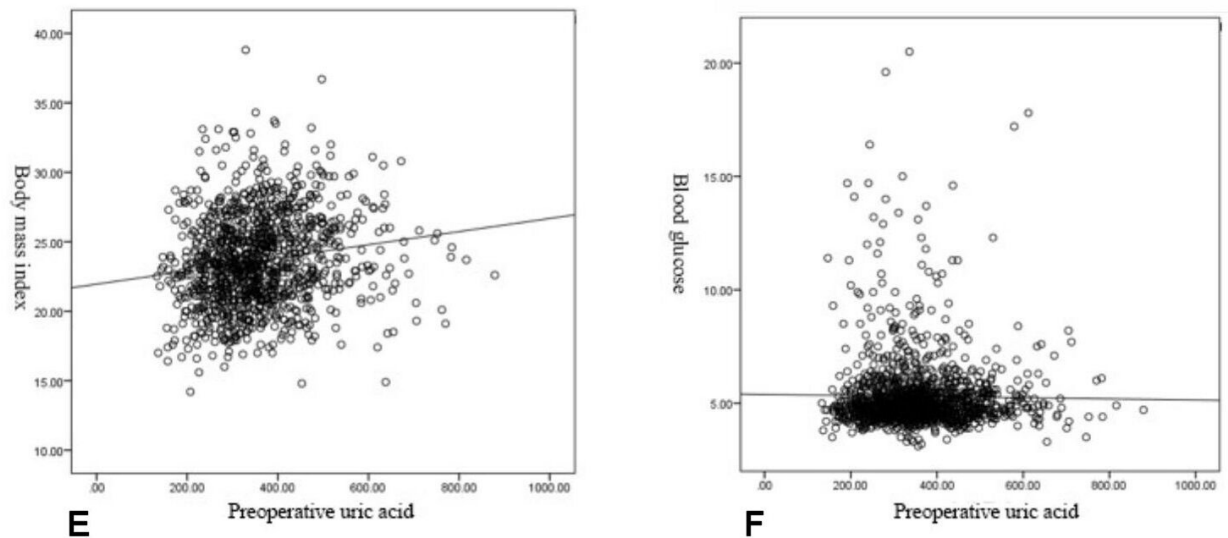

$$
\begin{aligned}
& r=0.157 \\
& P<0.001
\end{aligned}
$$$$
r=-0.016
$$$$
P=0.549
$$

Figure 1 Correlation of uric acid with blood lipid, body mass index and blood glucose. 


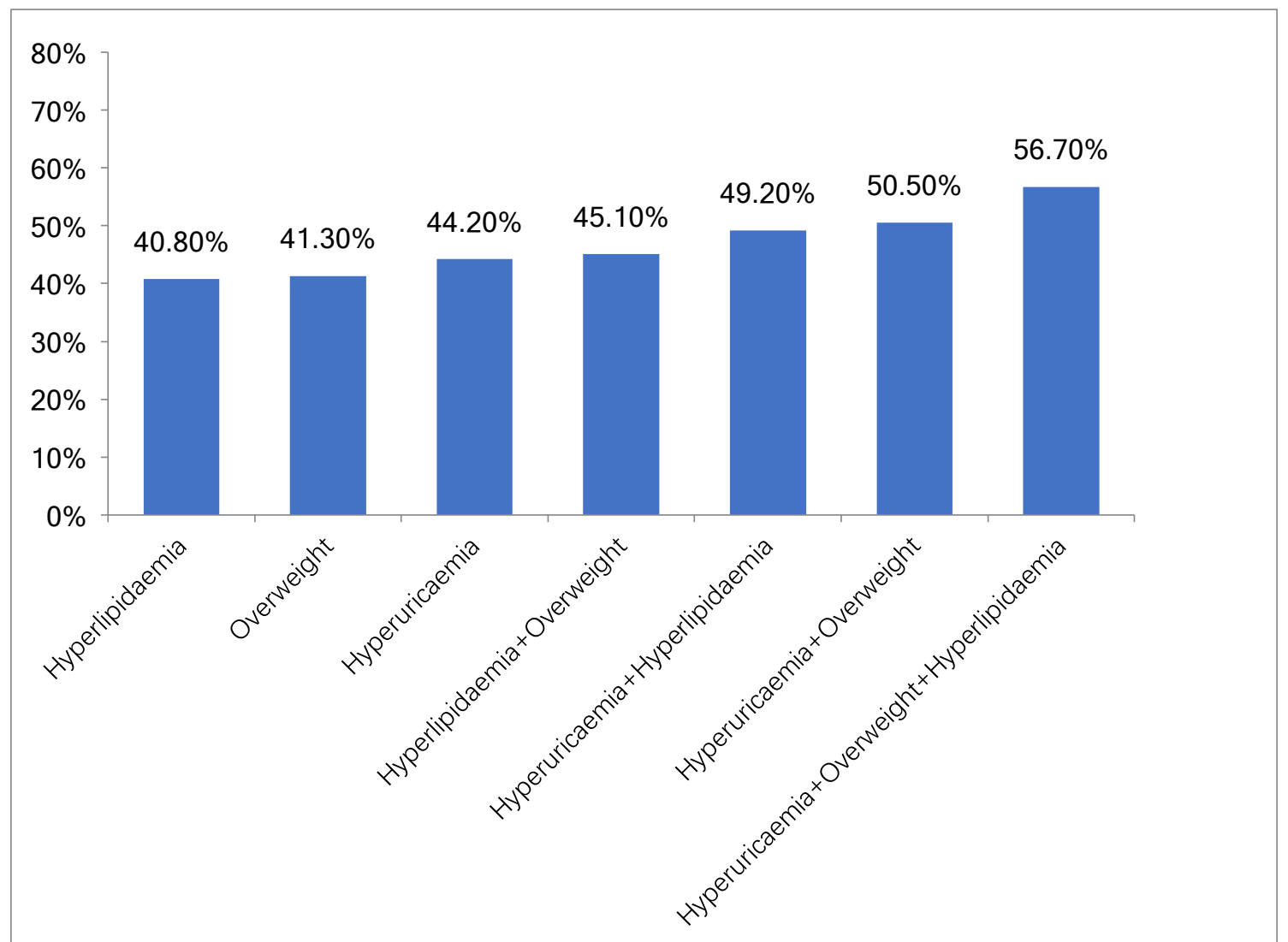

Figure 2 Comparison of acute kidney injury incidence among different subgroups.

respectively. The mechanism of hyperuricaemia caused by AKI is related to the crystallisation of uric acid. Urate crystals have a proinflammatory effect, which can cause renal tubular damage. Moreover, elevated serum uric acid may lead to endothelial dysfunction, oxidative stress and elevated $\mathrm{C}$ reactive protein. ${ }^{11}{ }^{18-22}$ Multiple studies have confirmed that hyperuricaemia is an independent risk factor for AKI. A retrospective study of 18444 hospitalised patients divided into four groups according to uric acid levels showed that blood uric acid of $>6.7 \mathrm{mg} / \mathrm{dL}$ (men) or $>5.4 \mathrm{mg} / \mathrm{dL}$ (women) correlated with a higher risk of AKI compared with blood uric acid of $<4.5 \mathrm{mg} / \mathrm{dL}$ (men) and $<3.6 \mathrm{mg} / \mathrm{dL}$ (women) with ORs of $3.2(95 \%$ CI 2.55 to 4.10$)$ in men $(p<0.001)$ and $3.1(95 \%$ CI 2.40 to $4.19)$ in women $(\mathrm{p}<0.001) .{ }^{8}$ Another retrospective study including 1536 patients with rheumatic heart disease showed that uric acid was independently associated with in-hospital $(\mathrm{OR}=1.21,95 \% \mathrm{CI}$ 1.06,1.37; $\mathrm{p}=0.004)$ and 1-year $(\mathrm{HR}=1.17,95 \%$ CI 1.05,1.29; $\mathrm{p}=0.003)$ mortality after valve replacement surgery. ${ }^{10}$ A prospective study by Gaipov et al showed that uric acid seemed to predict the progression of AKI and RRT requirement in patients who underwent cardiac surgery better than neutrophil gelatinase-associated lipocalin. ${ }^{9}$ A meta-analysis including 18 cohort studies with 75200 patients found that the hyperuricaemia group had a significantly higher risk of AKI compared with the controls (OR 2.24, 95\% CI 1.76 to $2.86 ; \mathrm{p}<0.01)$. The risk rate after percutaneous coronary intervention (PCI) was much higher in the hyperuricaemia group than in the control group ( $16.0 \%$ vs $5.3 \%$, OR $3.24,95 \%$ CI 1.93 to $5.45 ; \mathrm{p}<0.01){ }^{4}$ Similar findings on AKI and in-hospital mortality have been reported in Asian populations. ${ }^{23}$

Previous studies have confirmed that hyperuricaemia is closely related to lipid metabolism. ${ }^{24}$ Ali et al found that serum uric acid level was positively correlated with serum levels of TC, triglycerides and LDL. ${ }^{25}$ A study involving 536 patients undergoing radical gastrectomy concluded that age, BMI, hypertension, hyperlipidaemia and preoperative cystatin $\mathrm{C}$ level were independent risk factors for AKI. ${ }^{12}$ The results of our study showed that the incidence of hyperlipidaemia in the AKI group was higher than that in the non-AKI group (36.5\% vs $34 \%, \mathrm{p}=0.351)$. In addition, there was a positive correlation between uric 
Table 3 Univariate logistic analysis of risk factors for cardiac surgery-associated acute kidney injury

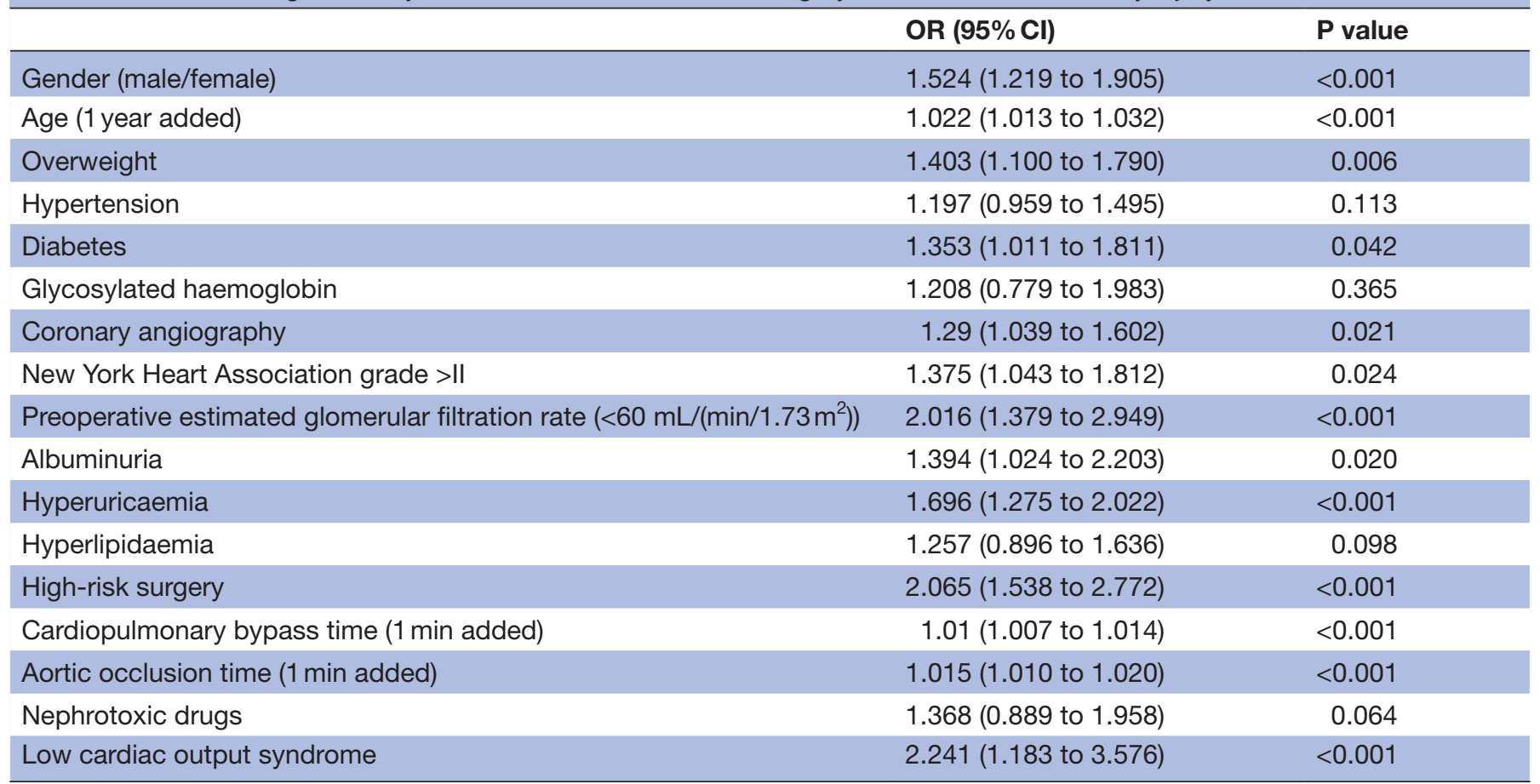

acid and triglyceride levels $(\mathrm{r}=0.221, \mathrm{p}<0.001)$ and $\mathrm{a}$ negative correlation between uric acid and HDL-c levels $(\mathrm{r}=0.190, \mathrm{p}<0.001)$. The possible mechanisms may be as follows: first, both purine synthesis and lipid metabolism are carried out in the liver, and hyperuricaemia causes increased glucose-6-phosphate activity, resulting in increased blood lipid synthesis from liver fatty acids; second, insulin resistance may lead to simultaneous elevation of uric acid and blood lipid (triglyceride and cholesterol) levels; third, long-term dyslipidaemia involves the afferent and efferent arterioles, impairs the glomerular filtration function and leads to hyperuricaemia due to the reduction of renal uric acid clearance. ${ }^{2627}$

In this study, there was a positive correlation between uric acid levels and BMI $(\mathrm{R}=0.157, \mathrm{p}<0.001)$; in univariate regression analysis, being overweight was an independent risk factor for $\mathrm{AKI}(\mathrm{OR}=1.314,95 \% \mathrm{CI} 1.031$ to 1.675 ; $\mathrm{p}=0.027)$. Obesity is significantly related to insulin resistance and hyperinsulinaemia, and obesity can activate the renin-angiotensi- aldosterone system; these can cause glomerular haemodynamic changes, such as glomerular hyperperfusion and hyperfiltration, lead to further glomerular injury and reduce the number of functional nephrons in obese patients, which can cause glomerulosclerosis. ${ }^{28-31}$ In addition, adipocytes may act as sites for activated inflammatory cytokines and oxidative stress, ${ }^{30} 32$ which may damage the kidneys. A single-centre cohort study involving 15470 critically ill patients found that each $5 \mathrm{~kg} / \mathrm{m}^{2}$ increase in BMI was associated with a $10 \%$ risk $(95 \%$ CI 1.06 to $1.24, \mathrm{p}<0.001)$ of more severe AKI. ${ }^{14}$ Previous studies have shown that the incidence of postoperative AKI in obese patients (BMI $\geq 28)$ increased significantly, and that BMI was an independent risk factor for
AKI. Another analysis involving 445 patients after cardiac surgery found that BMI was associated with an increased risk of AKI within 30 days, with the risk of AKI increasing by $26.5 \%$ for every $5 \mathrm{~kg} / \mathrm{m}^{2}$ increase in BMI. ${ }^{33}$

In the current study, multivariate logistic regression analysis found that hyperlipidaemia alone was not an independent risk factor for AKI, but the coexistence of hyperlipidaemia and being overweight was identified as a new risk factor. In the final model, the combination of hyperuricaemia, hyperlipidaemia and being overweight further increased the risk. The results show that hyperlipidaemia alone does not increase the risk of AKI, but in combination with other risk factors, such as being overweight or hyperuricaemia, it greatly increases the AKI risk. This means that controlling a single risk factor might not be effective, but controlling for multiple risk factors simultaneously may be sufficient to prevent AKI.

This study had several limitations. First, this was a single-centre retrospective analysis based on prospectively collected data. Second, the degree of hyperuricaemia was not graded, and the effect of uric acid-lowering treatment on the occurrence of CSA-AKI was not clear. Third, prospective studies should be conducted in the future to confirm the effects of urate-lowering therapy, fat reduction and weight loss therapy on the risk of AKI.

\section{CONCLUSIONS}

The incidence of AKI increased according to the uric acid quartiles, and patients with higher uric acid levels had higher proportions of hypertension, being overweight and hyperlipidaemia. The level of uric acid is related to blood lipids and BMI, respectively. Hyperuricaemia was 
Table 4 Multivariate regression analysis of risk factors for cardiac surgery-associated acute kidney injury

\begin{tabular}{|c|c|c|c|}
\hline & OR $(95 \% \mathrm{Cl})$ & $P$ value & $\begin{array}{l}\text { Hosmer-Lemeshow } \\
\text { test ( } \mathrm{P} \text { value) }\end{array}$ \\
\hline Model $1^{*}$ & & & 0.433 \\
\hline Male & 1.797 (1.378 to 2.344$)$ & $<0.001$ & \\
\hline Age & $1.026(1.014$ to 1.038$)$ & $<0.001$ & \\
\hline Diabetes & 1.681 (1.188 to 2.378$)$ & 0.003 & \\
\hline NYHA grade $>I I$ & $1.120(1.064$ to 1.437$)$ & 0.023 & \\
\hline Preoperative eGFR $\left(<60 \mathrm{~mL} /\left(\mathrm{min} / 1.73 \mathrm{~m}^{2}\right)\right)$ & $1.668(1.071$ to 2.598$)$ & 0.024 & \\
\hline High-risk surgery & 1.749 (1.117 to 2.380$)$ & $<0.001$ & \\
\hline Extracorporeal circulation time & $1.046(1.012$ to 2.362$)$ & $<0.001$ & \\
\hline Hyperuricaemia & 1.237 (1.095 to 1.885$)$ & 0.009 & \\
\hline Model 2 (model 1+overweightxhyperlipidaemia) $†$ & & & 0.638 \\
\hline Male & $1.892(1.415$ to 2.531$)$ & $<0.001$ & \\
\hline Age & $1.032(1.019$ to 1.046$)$ & $<0.001$ & \\
\hline Diabetes & $1.490(1.002$ to 2.215$)$ & 0.047 & \\
\hline Preoperative eGFR (<60 mL/(min/1.73m²)) & $1.741(1.150$ to 2.635$)$ & 0.029 & \\
\hline High-risk surgery & 1.698 (1.134 to 2.459$)$ & 0.013 & \\
\hline Extracorporeal circulation time & 1.009 (1.006 to 1.013$)$ & $<0.001$ & \\
\hline Overweightxhyperlipidaemia & 1.544 (1.059 to 2.252$)$ & 0.024 & \\
\hline Model 3 (model 1+hyperuricaemiaxoverweightxhyperlipidaemia)‡ & & & 0.597 \\
\hline Male & $1.658(1.303$ to 2.110$)$ & $<0.001$ & \\
\hline Age & 1.029 (1.017 to 1.040$)$ & $<0.001$ & \\
\hline Preoperative eGFR $\left(<60 \mathrm{~mL} /\left(\mathrm{min} / 1.73 \mathrm{~m}^{2}\right)\right)$ & 1.739 (1.165 to 2.595$)$ & 0.007 & \\
\hline High-risk surgery & 1.779 (1.128 to 2.673$)$ & 0.017 & \\
\hline Extracorporeal circulation time & $1.011(1.007$ to 1.015$)$ & $<0.001$ & \\
\hline Hyperuricaemia×overweight×hyperlipidaemia & $3.126(1.731$ to 5.646$)$ & $<0.001$ & \\
\hline
\end{tabular}

${ }^{*}$ Covariates adjusted for in model A: male, age, overweight, diabetes, angiography, NYHA grade $>$ II, preoperative eGFR $<60 \mathrm{~mL} /\left(\mathrm{min} / 1.73 \mathrm{~m}{ }^{2}\right)$, high-risk surgery, extracorporeal CPB time and aortic occlusion time.

†Covariates adjusted for in model B: male, age, overweight, diabetes, angiography, NYHA grade $>I I$, preoperative eGFR $<60 \mathrm{~mL} /\left(\mathrm{min} / 1.73 \mathrm{~m}^{2}\right)$, high-risk surgery, extracorporeal CPB time and aortic occlusion time.

$\ddagger$ Covariates adjusted for in model C: male, age, overweight, diabetes, angiography, NYHA grade $>$ Il, preoperative eGFR $<60 \mathrm{~mL} /\left(\mathrm{min} / 1.73 \mathrm{~m}^{2}\right)$, high-risk surgery, extracorporeal CPB time and aortic occlusion time.

CPB, cardiopulmonary bypass; eGFR, estimated glomerular filtration rate; NYHA, New York Heart Association.

an independent risk factor for CSA-AKI; the combination of being overweight and having hyperlipidaemia was an independent risk factor, but being overweight or having hyperlipidaemia alone was not. The combination of hyperuricaemia, being overweight and hyperlipidaemia further increases the risk of AKI. Therefore, reducing combinations of risk factors might reduce the risk of AKI. Continued studies are needed to focus on the early detection of, and intervention for, these and other risk factors.

\section{Author affiliations}

${ }^{1}$ Department of Nephrology, Xiamen Branch, Zhongshan Hospital, Fudan University, Xiamen, China

${ }^{2}$ Department of Nephrology, Shanghai Jing'an District Central Hospital, Shanghai, China

${ }^{3}$ Department of Nephrology, Zhongshan Hospital, Fudan University, Shanghai, China ${ }^{4}$ Department of Cardiovascular Surgery, Zhongshan Hospital, Fudan University, Shanghai, China
${ }^{5}$ Department of Critical Care Medicine, Zhongshan Hospital, Fudan University, Shanghai, China

Acknowledgements We express our sincere gratitude to our colleagues whose names do not appear on the paper but contributed diligently to the research. We thank Xin Chen, Yanting Shi, Zhihong Chen, Xueping Lin, Man Su and Yunlu Zhang for data collection.

Contributors JX, YS and HL designed the study; YS, YL, BS, WJ, CW, ZL, XD, JT and YW participated in the acquisition of data; YS, JX, YF and HL researched and evaluated the literature; YS, $X X$ and JX undertook the statistical analysis; $\mathrm{HL}$ wrote the first draft of the manuscript; YS, HL, JX, JT and YW made substantial contributions to the interpretation of data. JX is responsible for the overall content as guarantor. All authors approved the final manuscript for publication.

Funding The study was funded by Shanghai Municipal Hospital Frontier Technology Project, supported by Shanghai ShenKang Hospital Development Center (number SHDC12018127), National Natural Science Foundation of China (81901939), Xiamen Science and Technology Plan in 2018 (3502Z20184009) and Dynamic Alert System for Acute Kidney Injury and Multidisciplinary standard (3502Z20199179). 
Competing interests None declared.

Patient consent for publication Not applicable.

Ethics approval This study involves human participants and was approved by the ethical committee of Zhongshan Hospital, Fudan University (number B2017039). The study was conducted ethically in accordance with the World Medical Association Declaration of Helsinki. Participants gave informed consent to participate in the study before taking part.

Provenance and peer review Not commissioned; externally peer reviewed.

Data availability statement Data are available upon reasonable request via email to the corresponding author (xu.jiarui@zs-hospital.sh.cn).

Supplemental material This content has been supplied by the author(s). It has not been vetted by BMJ Publishing Group Limited (BMJ) and may not have been peer-reviewed. Any opinions or recommendations discussed are solely those of the author(s) and are not endorsed by BMJ. BMJ disclaims all liability and responsibility arising from any reliance placed on the content. Where the content includes any translated material, BMJ does not warrant the accuracy and reliability of the translations (including but not limited to local regulations, clinical guidelines, terminology, drug names and drug dosages), and is not responsible for any error and/or omissions arising from translation and adaptation or otherwise.

Open access This is an open access article distributed in accordance with the Creative Commons Attribution Non Commercial (CC BY-NC 4.0) license, which permits others to distribute, remix, adapt, build upon this work non-commercially, and license their derivative works on different terms, provided the original work is properly cited, appropriate credit is given, any changes made indicated, and the use is non-commercial. See: http://creativecommons.org/licenses/by-nc/4.0/.

\section{ORCID iD}

Yiqi Su http://orcid.org/0000-0003-1042-3878

\section{REFERENCES}

1 Lagny M-G, Jouret F, Koch J-N, et al. Incidence and outcomes of acute kidney injury after cardiac surgery using either criteria of the RIFLE classification. BMC Nephrol 2015;16:76-84.

2 Machado MN, Nakazone MA, Maia LN. Prognostic value of acute kidney injury after cardiac surgery according to kidney disease: improving global outcomes definition and staging (KDIGO) criteria. PLoS One 2014;9:e98028.

3 Bellomo R, Auriemma S, Fabbri A, et al. The pathophysiology of cardiac surgery-associated acute kidney injury (CSA-AKI). Int J Artif Organs 2008;31:166-78.

$4 \mathrm{Xu} \mathrm{X}, \mathrm{Hu}$ J, Song N, et al. Hyperuricemia increases the risk of acute kidney injury: a systematic review and meta-analysis. BMC Nephrol 2017;18:27-40.

5 Kaufeld T, Foerster KA, Schilling T, et al. Preoperative serum uric acid predicts incident acute kidney injury following cardiac surgery. BMC Nephrol 2018;19:161-5.

6 Zou Z, Zhuang Y, Liu L, et al. Role of body mass index in acute kidney injury patients after cardiac surgery. Cardiorenal Med 2017;8:9-17.

7 Nie S, Tang L, Zhang W, et al. Are there modifiable risk factors to improve AKI? Biomed Res Int 2017;2017:5605634

8 Kang MW, Chin HJ, Joo K-W, et al. Hyperuricemia is associated with acute kidney injury and all-cause mortality in hospitalized patients. Nephrology 2019;24:718-24.

9 Gaipov A, Solak Y, Turkmen K, et al. Serum uric acid may predict development of progressive acute kidney injury after open heart surgery. Ren Fail 2015;37:96-102.

10 Wei X-B, Jiang L, Liu Y-H, et al. Serum uric acid as a simple risk factor in patients with rheumatic heart disease undergoing valve replacement surgery. Clin Chim Acta 2017;472:69-74.

11 Ejaz AA, Johnson RJ, Shimada $M$, et al. The role of uric acid in acute kidney injury. Nephron 2019;142:275-83.
12 Zhang J, Feng G, Yang Y, et al. Acute kidney injury after radical gastrectomy: a single center study. Int Urol Nephrol 2014;46:973-7.

13 Barrett MP, Moore D, Smith FG, et al. The impact of body mass index on mortality in patients with acute kidney injury: a systematic review protocol. Syst Rev 2018;7:173-8.

14 Danziger J, Chen KP, Lee J, et al. Obesity, acute kidney injury, and mortality in critical illness. Crit Care Med 2016;44:328-34.

15 Kellum JA, Lameire N, KDIGO AKI Guideline Work Group. Diagnosis, evaluation, and management of acute kidney injury: a KDIGO summary (Part 1). Crit Care 2013;17:204-18.

16 Mancia G, Fagard R, Narkiewicz K. ESH/ESC practice guidelines for the management of arterial hypertension. Blood Press 2013;2014:3-16.

17 Zhou B-F, Cooperative Meta-Analysis Group of the Working Group on Obesity in China. Predictive values of body mass index and waist circumference for risk factors of certain related diseases in Chinese adults-study on optimal cut-off points of body mass index and waist circumference in Chinese adults. Biomed Environ Sci 2002;15:83-96.

18 Kanellis J, Watanabe S, Li JH, et al. Uric acid stimulates monocyte chemoattractant protein-1 production in vascular smooth muscle cells via mitogen-activated protein kinase and cyclooxygenase-2. Hypertension 2003;41:1287-93.

19 Kang D-H, Park S-K, Lee I-K, et al. Uric acid-induced C-reactive protein expression: implication on cell proliferation and nitric oxide production of human vascular cells. J Am Soc Nephrol 2005;16:3553-62.

20 Corry DB, Eslami P, Yamamoto K, et al. Uric acid stimulates vascular smooth muscle cell proliferation and oxidative stress via the vascular renin-angiotensin system. J Hypertens 2008;26:269-75.

21 Yu M-A, Sánchez-Lozada LG, Johnson RJ, et al. Oxidative stress with an activation of the renin-angiotensin system in human vascular endothelial cells as a novel mechanism of uric acid-induced endothelial dysfunction. J Hypertens 2010;28:1234-42.

22 Khosla UM, Zharikov S, Finch JL, et al. Hyperuricemia induces endothelial dysfunction. Kidney Int 2005;67:1739-42.

23 Park S-H, Shin W-Y, Lee E-Y, et al. The impact of hyperuricemia on in-hospital mortality and incidence of acute kidney injury in patients undergoing percutaneous coronary intervention. Circ $J$ 2011;75:692-7.

24 Thottam GE, Krasnokutsky S, Pillinger MH. Gout and metabolic syndrome: a Tangled web. Curr Rheumatol Rep 2017;19:60-7.

25 Ali N, Rahman S, Islam S, et al. The relationship between serum uric acid and lipid profile in Bangladeshi adults. BMC Cardiovasc Disord 2019;19:42-8.

26 Assmann G, Cullen P, Erbey J, et al. Plasma sitosterol elevations are associated with an increased incidence of coronary events in men: results of a nested case-control analysis of the prospective cardiovascular Münster (PROCAM) study. Nutr Metab Cardiovasc Dis 2006;16:13-21.

27 Karalis DG. Intensive lowering of low-density lipoprotein cholesterol levels for primary prevention of coronary artery disease. Mayo Clin Proc 2009;84:345-52.

28 Henegar JR, Bigler SA, Henegar LK, et al. Functional and structural changes in the kidney in the early stages of obesity. J Am Soc Nephrol 2001;12:1211-7.

29 Chagnac A, Weinstein T, Korzets A, et al. Glomerular hemodynamics in severe obesity. Am J Physiol Renal Physiol 2000;278:F817-22.

30 Pausova Z. From big fat cells to high blood pressure: a pathway to obesity-associated hypertension. Curr Opin Nephrol Hypertens 2006;15:173-8.

31 Hostetter TH, Olson JL, Rennke HG, et al. Hyperfiltration in remnant nephrons: a potentially adverse response to renal ablation. Am J Physiol 1981;241:F85-93.

32 Chander PN, Gealekman O, Brodsky SV, et al. Nephropathy in Zucker diabetic fat rat is associated with oxidative and nitrosative stress: prevention by chronic therapy with a peroxynitrite scavenger ebselen. J Am Soc Nephrol 2004;15:2391-403.

33 Billings FT, Pretorius M, Schildcrout JS, et al. Obesity and oxidative stress predict AKI after cardiac surgery. J Am Soc Nephrol 2012;23:1221-8. 